PROCEEDINGS OF THE

AMERICAN MATHEMATICAL SOCIETY

Volume 133, Number 3, Pages 633-642

S 0002-9939(04)07632-4

Article electronically published on October 7, 2004

\title{
SECANT VARIETIES OF GRASSMANN VARIETIES
}

\author{
M. V. CATALISANO, A. V. GERAMITA, AND A. GIMIGLIANO \\ (Communicated by Michael Stillman)
}

\begin{abstract}
We consider the dimensions of the higher secant varieties of the Grassmann varieties. We give new instances where these secant varieties have the expected dimension and also a new example where a higher secant variety does not.
\end{abstract}

\section{INTRODUCTION}

The problem of determining the dimensions of the higher secant varieties of the classically studied projective varieties has a long and interesting history. Recent work by J. Alexander and A. Hirschowitz (see 1], for example) completed a project that was underway for over 100 years (see [9, [10, and [11]) and confirmed the conjecture that, apart from the quadratic Veronese varieties and a (few) well-known exceptions, all the Veronese varieties have higher secant varieties of the expected dimension.

There has been no comparable success with Segre varieties (nor is there even a compelling conjecture) although there is much interest in this question - and not only among geometers. In fact, this particular problem is strongly connected to questions in representation theory, coding theory and algebraic complexity theory (see [3] for recent results as well as a summary of known results, and 2 for connections with complexity theory). In this paper we investigate this same problem for another family of classically studied varieties, namely the Grassmann varieties in their Plücker embeddings.

Denote by $G_{k, n+1}$ the Grassmannian of $k$-dimensional subspaces of a fixed $(n+1)$ dimensional vector space. When we consider $G_{k, n+1}$ embedded (by the Plücker embedding) in $\mathbb{P}^{N_{k}}\left(N_{k}=\left(\begin{array}{c}n+1 \\ k\end{array}\right)-1\right)$ we write it as $\mathbb{G}(k-1, n)$.

The dimensions of the higher secant varieties to the Grassmannians of lines in projective space are well known (and we give a simple proof of this known result in Section 2) but, to the best of our knowledge, little more can be found in the classical or modern literature about this problem (although, see the comments of Ehrenborg in [5], and [ $\underline{8}$ for connections with coding theory).

Received by the editors November 26, 2002 and, in revised form, October 2, 2003.

2000 Mathematics Subject Classification. Primary 14M15, 15 A75.

The first author was supported in part by MIUR funds.

The second author was supported in part by MIUR funds, and by the Natural Sciences and Engineering Research Council of Canada.

The third author was supported in part by the University of Bologna, funds for selected research topics, and by MIUR funds.

(C)2004 American Mathematical Society Reverts to public domain 28 years from publication 
Our main result is Theorem 2.1(ii), in which we give many new instances where $\mathbb{G}(k-1, n)$ has secant varieties of the expected dimension. In particular, when $k-1>1$ we find that all the chordal varieties to $\mathbb{G}(k-1, n)$ have the expected dimension.

In Section 3 we discuss our search for defective secant varieties to Grassmannians. We give new proofs that $\mathbb{G}(2,6)$ and $\mathbb{G}(2,8)$ have defective secant varieties and also find a new Grassmannian with defective secant varieties, namely $\mathbb{G}(3,7)$.

Finally, we state a few words concerning our methods. Our approach uses, as its first step, the fundamental observation of Terracini about secant varieties (the socalled Lemma of Terracini). This lemma converts the problem of determining the dimension of a (higher) secant variety into one of finding the dimension of a certain linear space. The second step in our approach is to identify this linear space (via an exterior algebra version of apolarity) with a graded piece of an intersection of homogenous "fat ideals" in an exterior algebra. The final step consists of calculating the dimension of the appropriate piece of this ideal.

\section{Preliminaries, Grassmannians and exterior algebras}

We recall a few elementary facts about exterior algebras and Grassmannians. Let $V$ be a finite-dimensional vector space over the field $K$ (we assume that char $K=0$ and $K$ is algebraically closed) and let $\operatorname{dim} V=n+1 . \wedge(V)$ denotes the exterior algebra of $V . \wedge(V)$ is a graded (non-commutative) ring with $(\bigwedge(V))_{k}=\wedge^{k} V$. It is well known that $\wedge^{k} V$ is a finite-dimensional vector space and that $\operatorname{dim} \wedge^{k} V=\left(\begin{array}{c}n+1 \\ k\end{array}\right)$. The elements of $\wedge^{k} V$ are called exterior $k$-vectors (and sometimes skew-symmetric tensors). An exterior $k$-vector $T$ is said to be decomposable if we can find vectors $v_{1}, \ldots, v_{k} \in V$ such that $T=v_{1} \wedge \ldots \wedge v_{k}$. We say that the exterior $k$-vector $T$ has $\wedge$-rank $=r$ if it can be written as a sum of $r$ (but no fewer) decomposable exterior $k$-vectors.

It is natural to ask the following two questions:

1) What is the least integer $D(k, n+1)$ so that every exterior $k$-vector in $\wedge^{k} V$ has $\wedge$-rank $\leq D(k, n+1)$ ?

2) What is the least integer $E(k, n+1)$ for which there is a dense subset $U \subset \wedge{ }^{k} V$ (dense in the Zariski topology) such that every exterior $k$-vector in $U$ has $\wedge$-rank $\leq E(k, n+1)$ ? We will call this number the typical rank (called the essential rank in [5]) of $\wedge^{k} V$ ?

Our main focus in this paper will be on Question 2), and related issues.

We first recall the coordinate description of the canonical multilinear, alternating map

$$
\nu_{k}: \underbrace{V \times \cdots \times V}_{k \text { times }}: \rightarrow \wedge^{k} V \quad \text { defined by }\left(v_{1}, \ldots, v_{k}\right) \rightarrow v_{1} \wedge \ldots \wedge v_{k} .
$$

Choosing an ordered basis $\left\{e_{0}, \ldots, e_{n}\right\}$ for $V$ and lexicographically ordering the $k$-subsets $\left\{i_{1}, \ldots, i_{k}\right\}$ of $\{0, \ldots, n\}$ we obtain an ordered basis $\left\{e_{i_{1}} \wedge \cdots \wedge e_{i_{k}} \mid 0 \leq\right.$ $\left.i_{1}<\cdots<i_{k} \leq n\right\}$ for $\wedge^{k} V$. With these choices the map $\nu_{k}$ can now be written $\nu_{k}\left(v_{1}, \ldots, v_{k}\right)=\sum_{\left\{i_{1}, \ldots, i_{k}\right\}} m_{i_{1}, \ldots, i_{k}} e_{i_{1}} \wedge \ldots \wedge e_{i_{k}}$ where $m_{i_{1}, \ldots, i_{k}}$ is the $k \times k$-minor of $M$, the matrix with row vectors $v_{1}, \ldots, v_{k}$, formed by the columns $i_{1}, i_{2}, \ldots, i_{k}$.

Elementary considerations (see [6]) show that $\nu_{k}$ can be defined on $G_{k, n+1}$ with image $\mathbb{G}(k-1, n)$ in $\mathbb{P}^{N_{k}}$. We denote the coordinates in $\mathbb{P}^{N_{k}}$ by $z_{i_{1}, \ldots, i_{k}}$ where the sets $\left\{i_{1}, \ldots, i_{k}\right\}$ are chosen as above. 
Remark. Once we choose a basis for $V$ there is a natural 1-1 correspondence between the associated bases of $\wedge^{k} V$ and $\wedge^{n+1-k} V$. It is well known that, if we use this correspondence as an identification, the equations defining the Plücker embeddings of $\wedge^{k} V$ and $\wedge^{n+1-k} V$ into $\mathbb{P}^{N_{k}}=\mathbb{P}^{N_{n+1-k}}$ are the same. Thus, for the remainder of this paper we will only consider the Grassmannians $G_{k, n+1}$ where $k \leq[(n+1) / 2]$.

Definition 1.1. Let $X \subseteq \mathbb{P}^{N}$ be a closed irreducible projective variety; the $s^{t h}$ (higher) secant variety of $X$ is the closure of the union of all linear spaces spanned by $s$ points of $X$. We will use $X^{s}$ to denote the $s^{t h}$ secant variety of $X$.

As is well known, if $\operatorname{dim} X=n$, then $\operatorname{dim} X^{s} \leq \min \{N, s n+s-1\}$. When we have equality here we say that $X^{s}$ has the expected dimension. If there is not equality we say that $X^{s}$ is defective. A measure of this deficiency is given by the quantity $\min \{N, s n+s-1\}-\operatorname{dim} X^{s}$.

Let us go back to the embedded Grassmannian, $\mathbb{G}(k-1, n) \subset \mathbb{P}^{N_{k}}$. Since this variety parameterizes the decomposable exterior $k$-vectors in $\wedge^{k} V$, its secant varieties $[\mathbb{G}(k-1, n)]^{s}$ can be viewed as the closure of the locus of exterior $k$-vectors having $\wedge$-rank $=s$. This gives another interpretation of the numbers $E(k, n+1)$ we introduced above. More precisely: let $V$ be a $K$-vector space with $\operatorname{dim}_{K} V=n+1$. Then

$$
E(k, n+1)=\min \left\{s \mid[\mathbb{G}(k-1, n)]^{s}=\mathbb{P}^{N_{k}}\right\} .
$$

Moreover, information about the dimension of $[\mathbb{G}(k-1, n)]^{s}$, for values of $s$ different from $E(k, n+1)$, will tell us about the stratification of $\mathbb{P}\left(\wedge^{k} V\right)$ with respect to $\wedge$ rank.

Before proceeding it is worthwhile to recall that the dimensions of all the secant varieties to the Grassmannian of 2-dimensional subspaces of an $(n+1)$-dimensional space, i.e. the dimensions of $[\mathbb{G}(1, n)]^{s}$, are known (e.g. see [12]). Also, some results on $E(k, n+1)$ (from a more algebraic and combinatorial point of view) can be found in [5].

One of the main tools we will use to find the dimensions of secant varieties is the famous Lemma di Terracini. We recall that lemma now.

Lemma (Terracini's Lemma). Let $X$ be an irreducible variety in $\mathbb{P}^{n}$ and $P_{1}, \ldots, P_{s}$ generic points of $X$. Let $T_{P_{i}}$ be the projectivized tangent space to $X$ at $P_{i}$ and $\left\langle T_{P_{1}}, \ldots, T_{P_{s}}\right\rangle$ the (projective) linear subspace of $\mathbb{P}^{n}$ spanned by the $T_{P_{i}}$. Then,

$$
\operatorname{dim} X^{s}=\operatorname{dim}\left\langle T_{P_{1}}, \ldots, T_{P_{s}}\right\rangle .
$$

In view of Terracini's Lemma, we need a good description of the tangent space to a point of $\mathbb{G}(k-1, n) \subset \mathbb{P}^{N_{k}}$. We do this by studying the differential of the map $\nu_{k}$ above.

Since it is immaterial which point of $\mathbb{G}(k-1, n)$ we consider, we consider the point $\nu_{k}(M), M=\left(I_{k} 0\right)$, where $I_{k}$ is the $k \times k$ identity matrix and 0 is the $k \times(n+1-k)$ matrix of zeroes.

The image of this point of the Grassmannian is the point $[1: 0: \ldots: 0]$ of the Plücker embedding in $\mathbb{P}^{N_{k}}$. This is a point in the affine piece of $\mathbb{P}^{N_{k}}$ which is the complement of the closed set $V\left(z_{0,1,2, \ldots, k-1}\right)$, i.e. it is the point in $\mathbb{A}^{N_{k}}$ whose affine coordinates are $(0,0, \ldots, 0)$.

The points of the Grassmannian, $G_{k, n+1}$, with image in this affine piece are all represented by matrices in row reduced echelon form of the type $\left(I_{k} A\right)$, where $A$ 
is any matrix of size $k \times(n+1-k)$. Thus, an affine version of the map $\nu_{k}$ can be described by

$$
\nu_{k}: \mathbb{A}^{k(n+1-k)} \longrightarrow \mathbb{P}^{N_{k}} \backslash V\left(z_{0,1,2, \ldots, k-1}\right) \simeq \mathbb{A}^{N_{k}}=\mathbb{A}^{\left(\begin{array}{c}
n+1 \\
k
\end{array}\right)-1}
$$

where, if $A$ is the $k \times(n+1-k)$ matrix that represents a point of $\mathbb{A}^{k(n+1-k)}$, then the coordinates of the image of $A$ are given by considering all the maximal minors of $\left(I_{k} A\right)$ except the first minor, i.e. the minor corresponding to the first $k$ columns.

We now want to compute the linear transformation $d \nu_{k}(M)$ explicitly. Since we know that $\mathbb{G}(k-1, n)$ is a smooth variety of dimension $k(n+1-k)$, we are not interested in the rank of this linear transformation (it has to be $k(n+1-k)$ ). Rather, what we need is an explicit description of the vectors in the image of this linear transformation.

Since $M=\left(I_{k} 0\right)$ corresponds to the origin of $\mathbb{A}^{k(n+1-k)}$, the tangent space to $M$ can be thought of as all the matrices $B$ of size $k \times(n+1-k)$. A curve in $\mathbb{A}^{k(n+1-k)}$ through $M$ with tangent vector $B$ at $M$ is given by the line $\lambda B$, where $\lambda \in K$. The image (under $\nu_{k}$ ) of a particular point on this line (call it $\lambda_{0} B$ ) is given by the $k \times k$ minors of the matrix $\left(I_{k} \lambda_{0} B\right)$ (not the first minor). The minors that involve all but one column of $I_{k}$ give us all the possible $\lambda_{0} b_{i, j}$, where $b_{i, j}$ runs through all the entries of $B$, while those minors that involve all but $r$ columns of $I_{k}$ give us $\lambda_{0}^{r}(*)$ where $*$ is some $r \times r$ minor of $B$.

Thus the image of $B$ under the differential $d \nu_{k}(M)$ is

$$
d \nu_{k}(M)(B)=\lim _{\lambda \rightarrow 0}(1 / \lambda)\left(\nu_{k}(\lambda B)-\nu_{k}(0)\right) .
$$

It is easy to see that, in the limit, we get the entries $b_{i, j}$, wherever there was a $\lambda b_{i, j}$, and a 0 wherever there was a $\lambda^{r}(*)$ for $r>1$.

Put another way, the affine cone over $T_{\nu_{k}(M)}(\mathbb{G}(k-1, n))$ is

$$
\begin{gathered}
\left\{\left(\ldots, c_{i_{1}, \ldots, i_{k}}, \ldots\right) \in \mathbb{A}^{\left(\begin{array}{c}
n+1 \\
k
\end{array}\right)} \mid \text { where } c_{i_{1}, \ldots, i_{k}}=0\right. \text { if more than one of } \\
\left.i_{1}, \ldots, i_{k} \text { is different from } 0,1, \ldots, k-1\right\},
\end{gathered}
$$

i.e. it is the vector subspace of $\wedge^{k}(V)$ generated by all the $e_{i_{1}} \wedge \ldots \wedge e_{i_{k}}$ where at least $(k-1)$ of the $i_{j}$ 's are in $\{0,1, \ldots, k-1\}$.

There is a natural apolarity in the exterior algebra $\Lambda(V)$, i.e. there is a perfect pairing $\wedge^{k} V \times \wedge^{n+1-k} V \longrightarrow \wedge^{n+1} V \simeq K$ induced by the multiplication in $\wedge(V)$. Thus, if $Y$ is any subspace of $\wedge^{k} V$, we can associate to $Y$ its perpendicular space $Y^{\perp} \subseteq \wedge^{n+1-k} V$ where

$$
Y^{\perp}:=\left\{w \in \wedge^{n+1-k} V \mid v \wedge w=0 \text { for all } v \in Y\right\} .
$$

Of course, $\left(Y^{\perp}\right)^{\perp}=Y$ and from standard facts of linear algebra we have

$$
\operatorname{dim}_{K} Y^{\perp}+\operatorname{dim}_{K} Y=\operatorname{dim}_{K} \wedge^{k} V=\operatorname{dim}_{K} \wedge^{n+1-k} V .
$$

Now, if we let $Y=T_{\nu_{k}(M)}(\mathbb{G}(k-1, n))$ (with $M$ as above), then $Y^{\perp}=\left\langle e_{j_{1}} \wedge \ldots \wedge e_{j_{n+1-k}}\right|$ at least two of $\left\{j_{1}, \ldots, j_{n+1-k}\right\}$ are in $\left.\{0,1, \ldots, k-1\}\right\rangle$.

Put another way,

$$
Y^{\perp}=\left[\left(e_{0}, \ldots, e_{k-1}\right)^{2}\right]_{n+1-k},
$$

i.e. $Y^{\perp}$ is the degree $n+1-k$ part of the square of the ideal of $\bigwedge(V)$ generated by $e_{0}, \ldots, e_{k-1}$. 
Since, quite generally, whenever $W_{1}, \ldots, W_{s}$ are subspaces of $\wedge^{k} V$ we have that $\left(W_{1}+\cdots+W_{s}\right)^{\perp}=W_{1}^{\perp} \cap \cdots \cap W_{s}^{\perp}$, we obtain, by applying Terracini's Lemma and the results just obtained above, that:

Proposition 1.2. Let $V$ be a vector space of dimension $n+1$, and let

$$
\mathbb{B}_{1}=\left\{v_{1,1}, \ldots, v_{k, 1}\right\}, \cdots, \mathbb{B}_{s}=\left\{v_{1, s}, \ldots, v_{k, s}\right\}
$$

be a collection of $s$ sets of $k$ generic vectors in $V$. Let $I_{j}=\left(v_{1, j}, \ldots, v_{k, j}\right) \subset \wedge(V)$, $j=1, \ldots, s$, and let

$$
W=\left(I_{1}^{2} \cap \ldots \cap I_{s}^{2}\right)_{n+1-k} .
$$

Then the dimension of $[\mathbb{G}(k-1, n)]^{s}$ is

$$
\operatorname{dim}_{K} W^{\perp}-1=\left[\left(\begin{array}{c}
n+1 \\
k
\end{array}\right)-\operatorname{dim}_{K} W\right]-1=\left[\left(\begin{array}{c}
n+1 \\
k
\end{array}\right)-1\right]-\operatorname{dim}_{K} W .
$$

If the subspaces $V_{i}$, spanned by the $\mathbb{B}_{i}$ above, are as "disjoint as possible", we expect that

$$
\operatorname{dim}_{K} W=\max \left\{\left(\begin{array}{c}
n+1 \\
k
\end{array}\right)-s[k(n+1-k)+1], 0\right\},
$$

i.e. that

$$
\operatorname{dim}[\mathbb{G}(k-1, n)]^{s}=\min \left\{\left(\begin{array}{c}
n+1 \\
k
\end{array}\right)-1, s[k(n+1-k)]+(s-1)\right\},
$$

which is what we called the expected dimension of $[\mathbb{G}(k-1, n)]^{s}$. So, if $W$ has the expected dimension, then so does $[\mathbb{G}(k-1, n)]^{s}$. Moreover, if $W$ has more than the expected dimension, then the difference is precisely what we called the deficiency of $[\mathbb{G}(k-1, n)]^{s}$.

Remark. Notice that as long as $k s \leq n+1$ we can choose the vectors $v_{i, j}$ to be part of a basis $\left\{e_{0}, \ldots, e_{n}\right\}$ of $V$. It is precisely this case we will consider in the next section.

\section{The "MONOMiAL" CASE}

In this section we will suppose that $k s \leq n+1$, and let $W=\left[\left(e_{0}, \ldots, e_{k-1}\right)^{2} \cap\right.$ $\left.\ldots \cap\left(e_{k s-k}, \ldots, e_{k s-1}\right)^{2}\right]_{n+1-k}$. By analogy with the case of ideals in the symmetric algebra of a free module, we call such ideals "monomial ideals" of the exterior algebra. Thus, we can view $W$ as the degree $n+1-k$ part of a monomial ideal (the intersection of $s$ squares of monomial ideals).

We have the following theorem:

Theorem 2.1. Let $V, n, k$ be as in the previous section. Then:

i) if $k=2$, then $[\mathbb{G}(1, n)]^{s}$ is defective for $s<E(2, n+1)=\left\lfloor\frac{n+1}{2}\right\rfloor$ with defectiveness $2 s(s-1)$;

ii) while if $k \geq 3$ and $k s \leq n+1$, then $[\mathbb{G}(k-1, n)]^{s}$ has the expected dimension.

Proof. The case $k=2$ is known (e.g. see [5] or also [12]) but we give the proof here for the sake of completeness and also because the "monomial ideal" approach makes it quite easy.

First, assume that $2 s \leq n+1$ and consider

$$
W=\left[\left(e_{0}, e_{1}\right)^{2} \cap\left(e_{2}, e_{3}\right)^{2} \cap \ldots \cap\left(e_{2 s-2}, e_{2 s-1}\right)^{2}\right]_{n-1} .
$$


Once we note that $\left(e_{i}, e_{j}\right)^{2}=\left(e_{i} \wedge e_{j}\right)$ we have

$$
W=\left(e_{0} \wedge e_{1} \wedge \cdots \wedge e_{2 s-2} \wedge e_{2 s-1}\right)_{n-1} .
$$

Thus, $W$ will trivially be $=\{0\}$ if and only if $n-1<2 s$. This immediately gives that $E(2, n+1)=\left\lfloor\frac{n+1}{2}\right\rfloor$.

When $n-1 \geq 2 s$, we get that a basis for $W$ is given by the decomposable exterior $(n-1)$-vectors of the form:

$$
e_{0} \wedge e_{1} \wedge \ldots \wedge e_{2 s-1} \wedge e_{\alpha_{1}} \wedge \ldots \wedge e_{\alpha_{t}}
$$

where $t=n-1-2 s$ and $\alpha_{1}, \ldots, \alpha_{t}$ can be any $t$ elements in $\{2 s, \ldots, n\}$. So,

$$
\operatorname{dim}_{K} W=\operatorname{dim}_{K}\left(\wedge^{t}\left\langle e_{2 s}, \ldots, e_{n}\right\rangle\right)=\left(\begin{array}{c}
n-2 s+1 \\
n-2 s-1
\end{array}\right)=\left(\begin{array}{c}
n-2 s+1 \\
2
\end{array}\right) .
$$

A simple computation shows that this is not the expected dimension for $W$ (the expected dimension is $\left(\begin{array}{c}n+1 \\ 2\end{array}\right)-s(2 n-1)$ in this case) and that the defectiveness is $\delta=2 s^{2}-2 s=2 s(s-1)$. This completes the proof of $i$ ).

As for $i i)$, let $k \geq 3$ and $k s \leq n+1$. Recall that we may always suppose that $k \leq(n+1) / 2$ (see Remark 2 before Definition 1.1).

Case 1. Suppose that $2 s>n+1-k$. In order for there to be a monomial $e_{i_{1}} \wedge \ldots \wedge e_{i_{n+1-k}}$ in $W$ we must have at least two of $\left\{i_{1}, \ldots, i_{n+1-k}\right\}$ from each of the $s$ subsets

$$
\{0, \ldots, k-1\},\{k, \ldots, 2 k-1\}, \cdots,\{k s-k, \ldots, k s-1\} .
$$

If $2 s>n+1-k$, this automatically gives $W=0$. It remains to check that, under the given hypothesis on $s, n$ and $k$, the expected dimension of $W$ is also 0 .

Observe that in order to have $k \geq 3, k s \leq n+1$ and $2 s>n+1-k$ we must have

$$
\frac{n+1-k}{2}<s \leq \frac{n+1}{k}
$$

This implies that

$$
k^{2}-k(n+1)+2(n+1)>0 .
$$

The discriminant of the quadratic expression in $(\ddagger)$ is $\Delta=(n+1)(n-7)$, so for $n<7$, the quadratic expression has the same sign for every $k$. Since the coefficient of $k^{2}$ is positive, this means that $(\ddagger)$ is always satisfied for such $n$.

The conditions $k \geq 3, n \leq 6, k s \leq 7$, and $2 k \leq 7$ give only one solution $(k=3, s=2, n=5)$ and for these parameters the expected dimension for $W$ is indeed 0 . This gives $E(3,6)=2$ and $[\mathbb{G}(2,5)]^{2}=\mathbb{P}^{14}$ as required.

When $n=7$, the only possibility for $k$ is 3 and there is no $s$ satisfying ( $\dagger \dagger$ ).

So, suppose that $n \geq 8$. The quadratic equation associated to ( $\ddagger)$ then has two distinct roots

$$
r_{1}=\frac{n+1}{2}-\frac{\sqrt{\Delta}}{2}<\frac{n+1}{2}+\frac{\sqrt{\Delta}}{2}=r_{2} .
$$

Thus $(\ddagger)$ can be satisfied only for $k \leq r_{1}$ and $k \geq r_{2}$. But, $r_{2}>\frac{n+1}{2}$, so we need only consider $k \leq r_{1}$.

Notice that, for $n>8, \frac{n+1}{2}-\frac{\sqrt{\Delta}}{2}<3$, so the only case left to consider is $n=8$ and $k=3$ and there is no $s$ satisfying $(\dagger \dagger)$ for those values of $n$ and $k$. This completes Case 1. 
Case 2. $2 s \leq n+1-k$. In this case $W$ is certainly $\neq 0$. Since $W$ is the degree $n+1-k$ part of a monomial ideal, to compute $\operatorname{dim}_{K} W$ it is enough to count all the monomials of degree $n+1-k$ that are NOT in $W$. These are the monomials $e_{\alpha_{1}} \wedge \ldots \wedge e_{\alpha_{n+1-k}}$ with the property that, when we choose $\alpha_{1}, \ldots, \alpha_{n+1-k}$ from $\{0,1, \ldots, n\}$ we must make sure that from at least one of the $s$ subsets

$$
\{0, \ldots, k-1\},\{k, \ldots, 2 k-1\}, \cdots,\{(s-1) k, \ldots, s k-1\}
$$

we have chosen either nothing or one index.

If we concentrate at first (say) on the subset $\{0, \ldots, k-1\}$, then we need to choose $n+1-k$ elements from $\{0, \ldots, n\}$ such that all $n+1-k$ are outside $\{0, \ldots, k-1\}$ or at most one is in $\{0, \ldots, k-1\}$.

Since there are exactly $n+1-k$ elements outside $\{0, \ldots, k-1\}$, we have only one choice if we choose nothing from $\{0, \ldots, k-1\}$. However, there are $n+1-k$ subsets of $\{k, \ldots, n\}$ consisting of $(n+1-k)-1$ elements and to those we can add any one of the $k$ elements in $\{0, \ldots, k-1\}$. This gives us a total of $1+k(n+1-k)$ choices. Since we can do this for each of the $s$ subsets of $k$ elements above, we get a total of $s[k(n+1-k)+1]$ choices, i.e. there are exactly $s[k(n+1-k)+1]$ monomials of degree $n+1-k$ outside $W$. Since this is precisely the expected codimension of $W$, the theorem is proved.

The previous result has the following immediate corollary:

Corollary 2.2. Let $V, n, k$ be as above, but suppose that $k \geq 3$. Then all the chordal varieties, $[\mathbb{G}(k-1, n)]^{2}$ have the expected dimension.

\section{SOME FINAL REMARKS}

Since there appears to be so little in the literature about secant varieties to Grassmannians (but lots of folklore), we would like to collect some scattered results we have found and record them in this section. This will include an (apparently) new example of a deficient variety.

First, let $m, n, d$ be three positive integers such that $m=n+d-1$. Let $V, W$ be vector spaces over $K$ such that $\operatorname{dim}_{K} V=n$ and $\operatorname{dim}_{K} W=m$. Let $\phi_{d}: \mathbb{P}^{n-1} \longrightarrow \mathbb{P}^{t}$ be the Veronese embedding (and so $t=\left(\begin{array}{c}m \\ d\end{array}\right)-1$ ). The Plücker embedding of $\wedge^{d} W$ is also in $\mathbb{P}^{t}$ since, as Ehrenborg pointed out in [5], there is a 1-1 correspondence between $d$-subsets of an $m$-set and $d$-multisubsets of an $n$-set.

This suggests that in looking for deficient Grassmannians we consider all the deficient Veronese varieties. We do that now.

Case 1. All the quadratic Veronese varieties are defective; i.e. when $d=2$ we can choose $n$ arbitrarily. What about the corresponding Grassmannians? For $m=4,5$ the Grassmannian of lines is not defective, for trivial reasons. However, it is the case that all the other Grassmannians of lines are defective. (See Theorem 2.1i).) This is hopeful.

Case 2. There are exactly four other defective Veronese varieties. They correspond to:

i) $d=4, n=3$ and hence $m=6$;

ii) $d=4, n=4$ and hence $m=7$;

iii) $d=3, n=5$ and hence $m=7$;

iv) $d=4, n=5$ and hence $m=8$. 
Now, $i)$ suggests we look at $\mathbb{G}(3,5)$. Since $\wedge^{4} K^{6} \simeq \wedge^{2} K^{6}$, this is indeed defective; it is (again) a Grassmannian of lines.

From $i i)$ we consider $\mathbb{G}(3,6)$. Since $\wedge^{4} K^{7} \simeq \wedge^{3} K^{7}$ we should check if $\mathbb{G}(2,6)$ is defective. The secant plane variety should fill $\mathbb{P}^{34}$, but it does not, as we will now show.

By our method, we have to determine $\operatorname{dim} Z$, where

$$
Z=\left[\left(e_{0}, e_{1}, e_{2}\right)^{2} \cap\left(e_{3}, e_{4}, e_{5}\right)^{2} \cap\left(e_{6}, v, w\right)^{2}\right]_{4}
$$

where $\left\{e_{0}, \ldots, e_{6}\right\}$ is a basis of $W$ and $v, w$ are generic vectors in $W$.

We can actually suppose that $v, w \in\left\langle e_{0}, \ldots, e_{5}\right\rangle$; in fact, if $v=e_{6}+v_{1}$ and $w=e_{6}+w_{1}$ we get

$$
\left(e_{6}, v, w\right)^{2}=\left(e_{6} \wedge v, e_{6} \wedge w, v \wedge w\right)=\left(e_{6} \wedge v_{1}, e_{6} \wedge w_{1}, v_{1} \wedge w_{1}\right)=\left(e_{6}, v_{1}, w_{1}\right)^{2} .
$$

Notice that

$$
Z^{\prime}=\left[\left(e_{0}, e_{1}, e_{2}\right)^{2} \cap\left(e_{3}, e_{4}, e_{5}\right)^{2}\right]_{4}=\left\langle e_{i_{1}} \wedge e_{i_{2}} \wedge e_{i_{3}} \wedge e_{i_{4}}\right\rangle_{4},
$$

with $i_{1}<i_{2} \in\{0,1,2\}, i_{3}<i_{4} \in\{3,4,5\}$. Thus, if there is something in $Z$, it must be of the form $v \wedge w \wedge \Gamma, \Gamma \in \wedge^{2}\left\langle e_{0}, \ldots, e_{5}\right\rangle$. Actually, by the genericity of $v, w$ we can suppose that $\left\langle e_{0}, \ldots, e_{5}\right\rangle=\left\langle v, w, e_{1}, \ldots, e_{4}\right\rangle$; hence we can consider $\Gamma \in\left\langle e_{1}, \ldots, e_{4}\right\rangle$.

We can even further suppose that $v=e_{0}+\ldots+e_{5}, w=\sum_{i=0}^{5} b_{i} e_{i}$. Then it is enough to insure that for each of the 6 monomials, $m$, in $\Gamma$, the summands of $v \wedge w \wedge m$ that are not in $Z^{\prime}$ are all $=0$. This gives us 6 linear equations in the $b$ 's whose coefficient matrix is:

$$
\left(\begin{array}{cccccc}
b_{3}-b_{0} & b_{0}-b_{2} & 0 & b_{1}-b_{0} & 0 & 0 \\
b_{4}-b_{0} & 0 & b_{0}-b_{2} & 0 & b_{1}-b_{0} & 0 \\
b_{5}-b_{0} & 0 & 0 & 0 & 0 & 0 \\
0 & 0 & 0 & 0 & 0 & b_{5}-b_{0} \\
0 & b_{5}-b_{4} & b_{3}-b_{5} & 0 & 0 & b_{5}-b_{1} \\
0 & 0 & 0 & b_{5}-b_{4} & b_{3}-b_{5} & b_{5}-b_{2}
\end{array}\right),
$$

a matrix of rank 5 . Hence $\operatorname{dim} Z=1$, i.e. $\mathbb{G}(2,6)$ is defective for secant $\mathbb{P}^{2}$ 's, with deficiency 1 .

Note. Apparently this example is well known, as we recently learned from J. Landsberg (private communication). We were unable to find a reference to it in the literature.

From $i$ ii $)$ we are again brought to consider $\mathbb{G}(2,6)$, which we have just done.

From $i v$ ) we should consider $\mathbb{G}(3,7)$. We now show that this is indeed a defective variety.

The Grassmannian $\mathbb{G}(3,7)$ lies in $\mathbb{P}^{69}$. Since $\operatorname{dim} \mathbb{G}(3,7)=16$, it follows that $\operatorname{dim}[\mathbb{G}(3,7)]^{2}=33$ (by Corollary 2.2) as expected. One expects $\operatorname{dim}[\mathbb{G}(3,7)]^{3}=50$, but we show that $\operatorname{dim}[\mathbb{G}(3,7)]^{3}=49$.

As above, it is enough to prove that $\operatorname{dim}_{K} W=20$, where if $H_{1}, H_{2}$ and $H_{3}$ are three generic subspaces of $K^{8}$, each of dimension 4 , with bases $\left\{v_{i 1}, v_{i 2}, v_{i 3}, v_{i 4}\right\}$, $i=1,2,3$, then

$$
W=\left[\left(v_{11}, v_{12}, v_{13}, v_{14}\right)^{2} \cap\left(v_{21}, v_{22}, v_{23}, v_{24}\right)^{2} \cap\left(v_{31}, v_{32}, v_{33}, v_{34}\right)^{2}\right]_{4}
$$

in the exterior algebra $\bigwedge K^{8}$.

We can assume that $H_{1}=\left\langle e_{0}, e_{1}, e_{2}, e_{3}\right\rangle$, where the $e_{i}$ are part of a standard basis for $K^{8}$. Consider $H_{3} \cap\left\langle H_{2}, e_{i}\right\rangle$ for $i=0,1,2,3$. These are one-dimensional 
subspaces of $H_{3}$, which we denote by $\left\langle w_{i}\right\rangle$. By the genericity of the subspaces we may suppose that $\left\{w_{0}, w_{1}, w_{2}, w_{3}\right\}$ is a basis for $H_{3}$. But $w_{i}=e_{i}+u_{i}$ for $u_{i} \in H_{2}$ and again, using the genericity, we can assume that $\left\{u_{0}, u_{1}, u_{2}, u_{3}\right\}$ is a basis for $H_{2}$. So, letting $\left\{e_{0}, \ldots, e_{7}\right\}$ be a basis for $K^{8}$, and using the considerations above, we can assume, without loss of generality, that

$H_{1}=\left\langle e_{0}, e_{1}, e_{2}, e_{3}\right\rangle, H_{2}=\left\langle e_{4}, e_{5}, e_{6}, e_{7}\right\rangle$ and $H_{3}=\left\langle e_{0}+e_{4}, e_{1}+e_{5}, e_{2}+e_{6}, e_{3}+e_{7}\right\rangle$.

Now a simple calculation, using Macaulay 2 [7], shows that $\operatorname{dim}_{K} W$ is indeed 20 , and we are done. In fact, a simple hand check shows that the 20 forms in this space are:

$$
\begin{aligned}
& e_{0} \wedge e_{1} \wedge e_{4} \wedge e_{5}, e_{0} \wedge e_{2} \wedge e_{4} \wedge e_{6}, e_{0} \wedge e_{3} \wedge e_{4} \wedge e_{7} \\
& e_{1} \wedge e_{2} \wedge e_{5} \wedge e_{6}, e_{1} \wedge e_{3} \wedge e_{5} \wedge e_{7}, e_{2} \wedge e_{3} \wedge e_{6} \wedge e_{7}
\end{aligned}
$$

the 12 forms

$$
e_{i} \wedge e_{i+4} \wedge\left(e_{j} \wedge e_{k+4} \pm e_{k} \wedge e_{j+4}\right), \quad i \neq j \neq k, \quad i, j, k \in\{0,1,2,3\}
$$

and the two forms

$\left(e_{0} \wedge e_{5} \pm e_{1} \wedge e_{4}\right) \wedge\left(e_{2} \wedge e_{7} \pm e_{3} \wedge e_{6}\right), \quad\left(e_{0} \wedge e_{6} \pm e_{2} \wedge e_{4}\right) \wedge\left(e_{1} \wedge e_{6} \pm e_{3} \wedge e_{5}\right)$.

This shows that $[\mathbb{G}(3,7)]^{3}$ has deficiency 1. A calculation (using Macaulay 2) shows that $[\mathbb{G}(3,7)]^{5}=\mathbb{P}^{69}$.

It is not hard to show that if we fix three general points $P, Q$, and $R$ on the Grassmannian in $\mathbb{P}^{69}$, then the linear system of hyperplanes that contain the tangent spaces $T_{P}$ and $T_{Q}$ and that also contain $R$ have exactly a fixed line in the tangent plane $T_{R}$. (This is the geometric reason why the secant planes to $\mathbb{G}(3,7)$ have deficiency 1.)

Hence, when we take a fourth point $S$ on $\mathbb{G}(3,7)$ and consider the linear system of hyperplanes on $\mathbb{P}^{69}$ that contain $T_{P}, T_{Q}$, and $T_{R}$ and that also contain $\mathrm{S}$, that system contains three fixed lines in $T_{S}$. We expect those lines to be linearly independent and hence that the 4 -secants to $\mathbb{G}(3,7)$ have deficiency 4. Calculations with Macaulay 2 seem to confirm that expectation.

J. Landsberg has pointed out that our result shows that $\mathbb{G}(3,7)$ is the only homogeneous variety known where the first secant deficiency doesn't occur at either the first or last possible step.

It would be tempting, at this point, to conjecture that inasmuch as we have exhausted the list of defective Veronese varieties, then we have also exhausted the list of defective Grassmannians! Indeed, we hoped that this might be so, but J. Landsberg informed us that he had a communication from M. Catalano-Johnson indicating that $\mathbb{G}(2,8)$ might also be defective. We now verify that M. CatalanoJohnson's suspicion was well founded.

Notice that, in view of Theorem $2.1 \mathrm{ii}$ ), the space of secant $\mathbb{P}^{2}$ 's has the correct dimension. We will now show that $[\mathbb{G}(2,8)]^{4}$ has dimension 73 (instead of 75 , as expected). The argument follows the same lines we used to find the deficiency of $[\mathbb{G}(3,7)]^{3}$. Following the discussion in $\S 2$ we need to find $\operatorname{dim}_{K} W$, where if $H_{i}, i=1, \ldots, 4$ are 4 generic 3 -dimensional subspaces of $K^{9}$ and a basis for $H_{i}$ is $\left\{v_{i 1}, v_{i 2}, v_{i 3}\right\}$, then

$$
W=\left[\bigcap_{i=1}^{4}\left(v_{i 1}, v_{i 2}, v_{i 3}\right)^{2}\right]_{6}
$$


It is easy to see that, with no loss of generality, we can assume the four subspaces are:

and

$$
H_{1}=\left\langle e_{0}, e_{1}, e_{2}\right\rangle, H_{2}=\left\langle e_{3}, e_{4}, e_{5}\right\rangle, H_{3}=\left\langle e_{6}, e_{7}, e_{8}\right\rangle
$$

$$
H_{4}=\left\langle e_{0}+e_{3}+e_{6}, e_{1}+e_{4}+e_{7}, e_{2}+e_{5}+e_{8}\right\rangle .
$$

Using the exterior algebra routines in Macaulay 2 we find that $\operatorname{dim}_{K} W=10$ and so the dimension of $[\mathbb{G}(2,8)]^{4}$ is 73 , as stated.

These last two examples suggested that we should check $\mathbb{G}(2,11)$ and $\mathbb{G}(3,11)$ as well. We have verified that $[\mathbb{G}(2,11)]^{5}$ and $[\mathbb{G}(3,11)]^{4}$ are not defective.

\section{REFERENCES}

[1] J.Alexander, A.Hirschowitz. Polynomial interpolation in several variables. J. of Alg. Geom. 4 (1995). 201-222. MR.1311347 (96f:14065)

[2] P. Bürgisser, M. Clausen, M.A. Shokrollahi, Algebraic Complexity Theory, Vol. 315, Grund. der Math. Wiss., Springer, 1997. MK1440179 (99c:68002)

[3] M.V.Catalisano, A.V.Geramita, A.Gimigliano. Ranks of tensors, Secant Varieties of Segre Varieties and Fat Points. Lin. Algebra and Appl. 355 (2002). 263-285. MR1930149 (2003g:14070)

[4] M.V.Catalisano, A.V.Geramita, A.Gimigliano. Erratum of the Publisher to: "Ranks of tensors, Secant Varieties of Segre Varieties and Fat Points". Lin. Algebra and Appl. 367 (2003). 347-348. MF.1976931

[5] R.Ehrenborg. On Apolarity and Generic Canonical Forms. J. of Algebra 213 (1999). 167-194. MR 1674676 (2000a:15050)

[6] J. Harris, Algebraic Geometry, a first course, Springer, New York, 1993. MR1182558 (93j:14001)

[7] D.R.Grayson, M.E.Stillman, Macaulay 2, a software system devoted to supporting research in algebraic geometry, www.math.uiuc.edu .

[8] D. Yu. Nogin. Spectrum of codes associated with the Grassmannian G(3,9). Problems of Information Transmission, 33 (1997). 114-123. MR1663924 (2000e:94066)

[9] F.Palatini. Sulle varietà algebriche per le quali sono di dimensione minore dell' ordinario, senza riempire lo spazio ambiente, una o alcuna delle varietà formate da spazi seganti. Atti Accad. Torino Cl. Scienze Mat. Fis. Nat. 44 (1909). 362-375.

[10] A.Terracini. Sulle $V_{k}$ per cui la varietà degli $S_{h}(h+1)$-seganti ha dimensione minore dell'ordinario. Rend. Circ. Mat. Palermo 31 (1911). 392-396.

[11] W.Wakeford. On canonical forms. Proc. London Math. Soc. 18 (1919/20). 403-410.

[12] F.L.Zak. Tangents and Secants of Algebraic Varieties. Translations of Math. Monographs, vol. 127 AMS. Providence (1993). MR1234494 (94i:14053)

DiPEM, Facoltá di Ingegneria, Università di Genova, Italy

E-mail address: catalisano@dipem.unige.it

Dipartimento di Matematica, Università di Genova, Italy - and - Department of Mathematics and Statistics, Queens' University, Kingston, Ontario, Canada

E-mail address: geramita@dima.unige.it

Dipartimento di Matematica and CiRAM, Università di Bologna, Italy

E-mail address: gimiglia@dm.unibo.it 